\title{
Weak Gravity Conjecture in an Accelerating Universe
}

\author{
Ignatios Antoniadis ${ }^{1,2}$ and Karim Benakli ${ }^{1}$ \\ ${ }^{1}$ Laboratoire de Physique Théorique et Hautes Energies (LPTHE), UMR 7589 Sorbonne Université and CNRS, \\ 4 place Jussieu, 75252 Paris Cedex 05, France \\ ${ }^{2}$ Institute for Theoretical Physics, KU Leuven, Celestijnenlaan 200D, B-3001 Leuven, Belgium
}

\begin{abstract}
The study of de Sitter Reissner-Nordstrøm black holes allows us to uncover a Weak Gravity Conjecture in de Sitter space. It states that for a given mass $m$ there should be a state with a charge $q$ bigger than a minimal value $q_{\min }(m, l)$, depending on the mass and the de Sitter radius $l$, in Planck units. This reproduces the well known flat space-time result $q>m / \sqrt{2}$ in the large radius limit (large $l$ ). In the highly curved de Sitter space (small $l$ ) $q_{\min }$ behaves as $\sqrt{m l}$. Finally, we discuss the case of backgrounds from gauged R-symmetry in $N=1$ supergravity. This talk is based on 2006.12512 [1].
\end{abstract}

Keywords: de Sitter, black holes, swampland

DOI: 10.31526/ACP.BSM-2021.38

\section{INTRODUCTION}

One of the swampland conjectures [2], the Weak Gravity Conjecture (WGC) [3], states that for a $U(1)$ gauge symmetry with gauge coupling $g$, there is a least one state that has a charge $q$ bigger than its mass $m$, measured in Planck units, $8 \pi G=\kappa^{2}=1$ :

$$
m<\sqrt{2} g q
$$

This has a consequence that all black holes are able to decay without leaving remnants [3].

On the other hand, present cosmological observations seem to indicate a non-vanishing positive vacuum energy. Thepossible identification of the latter with a cosmological constant is theoretically challenging since as today attempts to construct de Sitter solutions in string theory have all failed (see for example [4,5]). A conjecture stating that such a space-time is absent in a consistent theory of gravity has been put forward [6]. It is based on arguments which may not hold at the string quantum level [7]. For a description of the observable Universe, de Sitter space could arise as an "approximate" background of some consistent string theory vacua.

Our aims is to find how the WGC is modified in the presence of a positive vacuum energy such that in the limit of flat spacetime one recovers (1). Some authors have considered the WGC in de Sitter [8,9], but as their constraint does not involve mass of WGC-states and it is clear if there is any obscure way in which they connect to (1). We live in a universe with a non-vanishing vacuum energy and daily use flat space-time laws having an impressive good agreement with experiments. This can be taken as a strong indication that when the vacuum energy is taken to be negligible, flat space-time laws such as (1) should be recovered.

We will use the de Sitter Reissner-Nordstrøm black hole extremal solutions, as discussed by [11] in order to derive our conjecture in de Sitter background (dS-WGC).

\section{THE RELEVANT PARAMETERS OF THE PROBLEM}

The line element in the de Sitter Reissner-Nordstrøm black hole [10] in Schwarzschild coordinates $(t, r, \theta, \phi)$ reads:

$$
d s^{2}=-f(r) d t^{2}+\frac{d r^{2}}{f(r)}+r^{2}\left(d \theta^{2}+\sin ^{2} \theta d \phi^{2}\right)
$$

with $f(r)$ is given by:

$$
f(r)=1-\frac{2 G m}{r}+\frac{G g^{2} q^{2}}{4 \pi r^{2}}-\frac{\Lambda}{3} r^{2},
$$

where $G$ is the Newton constant, $\Lambda$ is the cosmological constant, $g$ is the $U(1)$ gauge coupling, $g q$ is the black hole charge and $m$ its mass $(\hbar=c=1)$. This is solution of the equations of motion from the four-dimensional Einstein-Maxwell action

$$
S=\int d^{4} x \sqrt{-g}\left[\frac{1}{16 \pi G}(R-2 \Lambda)+\frac{1}{4 g^{2}} F^{\mu v} F_{\mu v}\right]
$$

where $F^{\mu v}$ is the electromagnetic field strength tensor with , $R$ is the Ricci scalar. 
It appears to be more convenient to use the quantities $M, Q, l$ with dimension of length:

$$
G m=\frac{\kappa^{2} m}{8 \pi}=M \quad, \quad \frac{G g^{2} q^{2}}{4 \pi}=\frac{\kappa^{2} g^{2} q^{2}}{32 \pi^{2}}=Q^{2}, \quad \Lambda=\frac{3}{l^{2}},
$$

where $\kappa^{2}=8 \pi G$ is the gravitational coupling, then:

$$
f(r)=1-\frac{2 M}{r}+\frac{Q^{2}}{r^{2}}-\frac{r^{2}}{l^{2}}
$$

It is useful to note that

$$
\frac{M^{2}}{Q^{2}}=\frac{\kappa^{2}}{2} \frac{m^{2}}{g^{2} q^{2}}
$$

is the ratio constrained by the WGC.

The case with both electric $Q$ and magnetic $Q_{m}$ charges is obtained through the substitution $Q^{2} \rightarrow Q^{2}+Q_{m}^{2}$ with

$$
Q_{m}^{2}=q_{m}^{2} g_{m}^{2}, g_{m}^{2}=\frac{4 \pi^{2}}{g^{2}}
$$

where $g_{m}$ is the gauge coupling and $q_{m}$ is the charge in the dual magnetic theory .

\section{REISSNER-NORDSTRøM WITH $\Lambda=0$ AND $Q \neq 0$ CASE}

We start by the case of $\Lambda=0$ and $Q \neq 0$ and briefly review a nowadays very well known result.

The metric has two horizons given by the locus of the zeros of

$$
f(r)=1-\frac{2 M}{r}+\frac{Q^{2}}{r^{2}}
$$

They correspond to the (inner) Cauchy horizon $r_{-}$and (outer) event horizon $r_{+}$, with:

$$
r_{-}=M\left(1-\sqrt{1-\frac{Q^{2}}{M^{2}}}\right) \quad, \quad r_{+}=M\left(1+\sqrt{1-\frac{Q^{2}}{M^{2}}}\right)
$$

The cancellation amounts to an equilibrium between the different terms in $f(r)$. The repulsive electromagnetic energy density gives rise to the positive $Q^{2}$ term while the attractive gravitational interaction leads to the negative $-2 M$. For $Q^{2}<M^{2}$, balancing against each other the two interactions, leads splitting the space-time patch described by the Schwarzschild coordinates into three regions: $0<r<r_{-}, r_{-}<r<r_{+}$and $r_{+}<r$. We thus have three cases:

- For $Q^{2}<M^{2}$, balancing against each other the two interactions splits the space-time patch, described by the Schwarzschild coordinates $t, r$, into three regions: $0<r<r_{-}, r_{-}<r<r_{+}$and $r_{+}<r$. We note that $t$ is space-like in the region $r r_{-}<r<r_{+}$ and time-like outside.

- $Q^{2}=M^{2}$, the two horizons are degenerate, the black hole temperature vanishes thus the black hole is extremal. This can be seen as the point where the gravitational attraction and electric repulsion have the same strength.

- $Q^{2}>M^{2}$, there is no horizon as the two roots $r_{ \pm}$are complex. The metric (11) exhibits a naked singularity at the origin $r=0$. One then can see that imposing $Q^{2}>M^{2}$ insures that the repulsive electromagnetic interaction is stronger than the attractive gravity and charged states that can not be black holes, because of the Weak Cosmic Censorship, are present in the theory.

This reproduces the $Q^{2}>M^{2}$ condition giving the "Weak Gravity Conjecture". In an asymptotically flat space-time, it allows extremal charged black holes to decay and avoids the presence of remnants when black holes decay and evaporate through Hawking radiation. However, it can also be obtained by other ways, for instance by computing the Newton force between two charged massive particles and compare it to their repulsive interaction.

\section{SCHWARZSCHILD BLACK HOLE IN DE SITTER SPACE-TIME}

In Schwarzschild coordinates $(t, r, \theta, \phi)$, the line element takes the form:

$$
d s^{2}=-f(r) d t^{2}+\frac{d r^{2}}{f(r)}+r^{2}\left(d \theta^{2}+\sin ^{2} \theta d \phi^{2}\right)
$$


where:

$$
f(r)=1-\frac{2 M}{r}-\frac{r^{2}}{l^{2}},
$$

thus the locus of the apparent horizons are obtained by solving:

$$
P_{0}(r) \equiv-l^{2} r f(r)=r^{3}-r l^{2}+2 M l^{2} \equiv\left(r-r_{--}\right)\left(r-r_{+}\right)\left(r-r_{C}\right)=0 .
$$

The sum of the roots vanishes and their product is negative. Therefore, one of the roots, we denote as $r_{--}$, is negative. It plays no role in the following analysis and the range of its values will not be discussed. The other two are either both real and positive, in which case we choose them such as $r_{+} \leq r_{C}$, or both complex. The real values of $r_{+}$and $r_{C}$ will describe the location of the event and cosmological horizons, respectively. They are given by:

$$
\begin{aligned}
r_{--} & =-\frac{e^{i \frac{\pi}{3}} l^{4 / 3}}{\sqrt{3}\left(-\sqrt{27} M+\sqrt{27 M^{2}-l^{2}}\right)^{1 / 3}}-\frac{e^{-i \frac{\pi}{3}} l^{2 / 3}\left(-\sqrt{27} M+\sqrt{27 M^{2}-l^{2}}\right)^{1 / 3}}{\sqrt{3}}-\frac{e^{i \frac{\pi}{3}} l^{2 / 3}\left(-\sqrt{27} M+\sqrt{27 M^{2}-l^{2}}\right)^{1 / 3}}{\sqrt{3}} \\
r_{+} & =-\frac{e^{-i \frac{\pi}{3}} l^{4 / 3}}{\sqrt{3}\left(-\sqrt{27} M+\sqrt{27 M^{2}-l^{2}}\right)^{1 / 3}} \\
r_{C} & =\frac{l^{4 / 3}}{\sqrt{3}\left(-\sqrt{27} M+\sqrt{27 M^{2}-l^{2}}\right)^{1 / 3}}+\frac{l^{2 / 3}\left(-\sqrt{27} M+\sqrt{27 M^{2}-l^{2}}\right)^{1 / 3}}{\sqrt{3}} .
\end{aligned}
$$

Figure 1 shows the dependence of $r_{+}$and $r_{C}$ as one varies of $M / l=m G / l$ in the region where they take real values, $M^{2} \leq l^{2} / 27$. In fact, one can discuss the three cases:

- $M^{2}<\frac{l^{2}}{27}$, where the two roots, $r_{+}<r_{C}$, are positive. One has a black hole with two horizons, the event horizon at $r_{+}$and the cosmological horizon at $r_{C}$. They define a region $0<r<r_{+}$where coordinate $t$ becomes space-like.

- The extremal case: $M^{2}=\frac{l^{2}}{27}$, the two horizons are degenerate. As the two horizons approach each other, one obtains a Nariai black hole and new coordinates have to be used in order to describe it. When they coincide, the black hole temperature vanishes. The attractive effect of the mass is exactly balanced by the repulsive effect of the cosmological constant.

- $M^{2}>\frac{l^{2}}{27}$, there is no real positive root. One observes that $f(r)$ is negative and that the curvature blows up at $r=0$. There is a naked singularity, a very massive black hole seems to have absorbed the local de Sitter patch [13]. This is again the situation which is not allowed by the Weak Cosmic Conjecture.

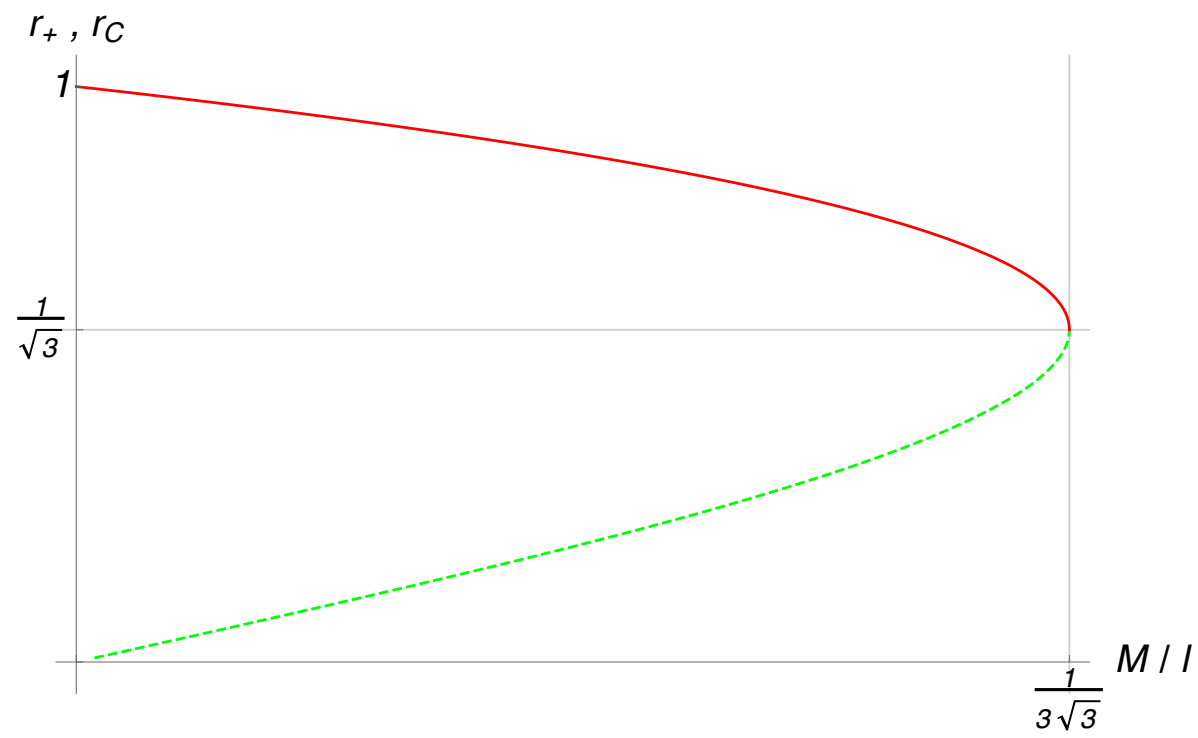

FIGURE 1: The positive roots of $P_{0}(r)$ : the red curve corresponds to the cosmological horizon and the dashed green one to the event horizon. As $M$ approaches its maximal allowed value $M=\frac{l}{\sqrt{27}}$, the two roots coincide. This corresponds to a neutral Nariai black hole. 


\section{CHARGED $Q \neq 0$ BLACK HOLE IN DE SITTER $\Lambda \neq 0$ :}

We turn now to the case of interest here: Reissner-Nordstrøm black holes in de Sitter background $l^{2}>0$ and look for the loci of the horizons, $r_{--}, r_{-}, r_{+}$and $r_{C}$ where:

$$
f(r)=1-\frac{2 M}{r}+\frac{Q^{2}}{r^{2}}-\frac{r^{2}}{l^{2}}=0 .
$$

In order to understand the nature and values of the latter as we vary the parameters $M, Q, l$ in (17), we study are roots of the quartic polynomial:

$$
P(r) \equiv-r^{2} f(r)=l^{-2} r^{4}-r^{2}+2 M r-Q^{2}
$$

The product of the four roots is negative, given by $-Q^{2}<0$. Thus two roots are real with opposite signs while the other two are either real of the same sign or complex conjugate. This depends on the sign of the discriminant $\Delta$ :

$$
\Delta=\frac{16}{l^{6}}\left(-\frac{27}{l^{2}}(M l)^{4}+\left(l^{2}+36 Q^{2}\right)(M l)^{2}-Q^{2}\left(l^{2}+4 Q^{2}\right)^{2}\right) .
$$

We choose $r_{--}<0$ to be the negative's root, and one to be the positive real one. For the other two roots, we have three possibilities:

1. $\Delta<0$, It is easy to see that two roots are complex and conjugate, one is positive: there is therefore only one $r_{-}=r_{+}^{*}$ (or $r_{-}>0$ and $r_{C}=r_{+}^{*}$ complex). There is only one horizon.

2. $\Delta>0$, we choose to order the positive roots such as that $0<r_{-}<r_{+}<r_{C}$. Then $r_{-}$and $r_{+}$are the inner and outer horizons, respectively. The (inner) Cauchy is probably unstable and addition of matter at the classical or quantum level. The outer horizon is the event horizon of the Reissner-Nordstrøm black hole and $r=r_{C}$ surface is the cosmological horizon of the de Sitter space.

3. $\Delta=0$, two horizons coincide $r_{-}=r_{+}$or $r_{+}=r_{C}$ and we are left with two apparent horizons.

These three cases are obtained by varying the value of the the parameter $M^{2} l^{2}$. In fact, $\Delta$ is a quadratic polynomial of $M^{2} l^{2}$. Considering its discriminant:

$$
\delta=\frac{256\left(l^{2}-12 Q^{2}\right)^{3}}{l^{14}}
$$

we can see that the roots of $\Delta$ are given by:

$$
\begin{aligned}
& M_{-}^{2}\left(l, Q^{2}\right)=\frac{1}{54 l}\left[l\left(l^{2}+36 Q^{2}\right)-\left(l^{2}-12 Q^{2}\right)^{3 / 2}\right] \\
& M_{+}^{2}\left(l, Q^{2}\right)=\frac{1}{54 l}\left[l\left(l^{2}+36 Q^{2}\right)+\left(l^{2}-12 Q^{2}\right)^{3 / 2}\right]
\end{aligned}
$$

and $\Delta$ is positive between and negative outside these roots (for $l^{2} \geq 12 Q^{2}$ ).

The horizon locus $r_{-}, r_{+}$and $r_{C}$ split the local patch of de Sitter in four regions:

- Region I: $0<r \leq r_{-}$where the repulsion of the electromagnetic interaction due to the charge $Q$, with a little help from the cosmological constant, dominates the gravitational attraction due to the mass $M$.

- Region II: $r_{-} \leq r \leq r_{+}$corresponds to the interior of the black hole as $r_{-}$and $r_{+}$are the Cauchy and event horizons, respectively. The gravity attraction, as seen from the negative term $-2 M / r$, is the dominant interaction here.

- Region III: $r_{+} \leq r \leq r_{C}$ where $t$ is time-like $(f(r)>0)$ as the constant term in $f(r) \sim 1+\cdots$ is dominant.

- Region IV: $r>r_{C}$ where the coordinate $t$ is space-like $(f(r)<0)$ due to the term $r^{2} / l^{2}$ becoming dominant, $f(r) \sim-r^{2}$. The cosmological constant leads to a repulsive interaction that rules over all the other interactions.

We have seen that (21) defines extreme values of the mass $M$ such that separate regions with different numbers of apparent horizons. It is helpful for the following discussion to define the notation $Q_{ \pm}$for the charges where for given fixed $M$ :

$$
M_{-}^{2}\left(l, Q_{-}^{2}\right)=M^{2}, M_{+}^{2}\left(l, Q_{+}^{2}\right)=M^{2},
$$

where, for convenience, we have chosen $Q$ positive.

We have plotted in Fig. 2 the functions $M_{ \pm}^{2}$ in the plane (mass, charge) of the black hole normalized as $M^{2} / l^{2}$ versus $Q^{2} / l^{2}$. For fixed $M$, we can separate the different cases:

1. $M^{2}<\frac{l^{2}}{27}: Q_{+}$does not exist (see the lower horizontal blue line in Fig. 2). As $Q \nearrow$ : 


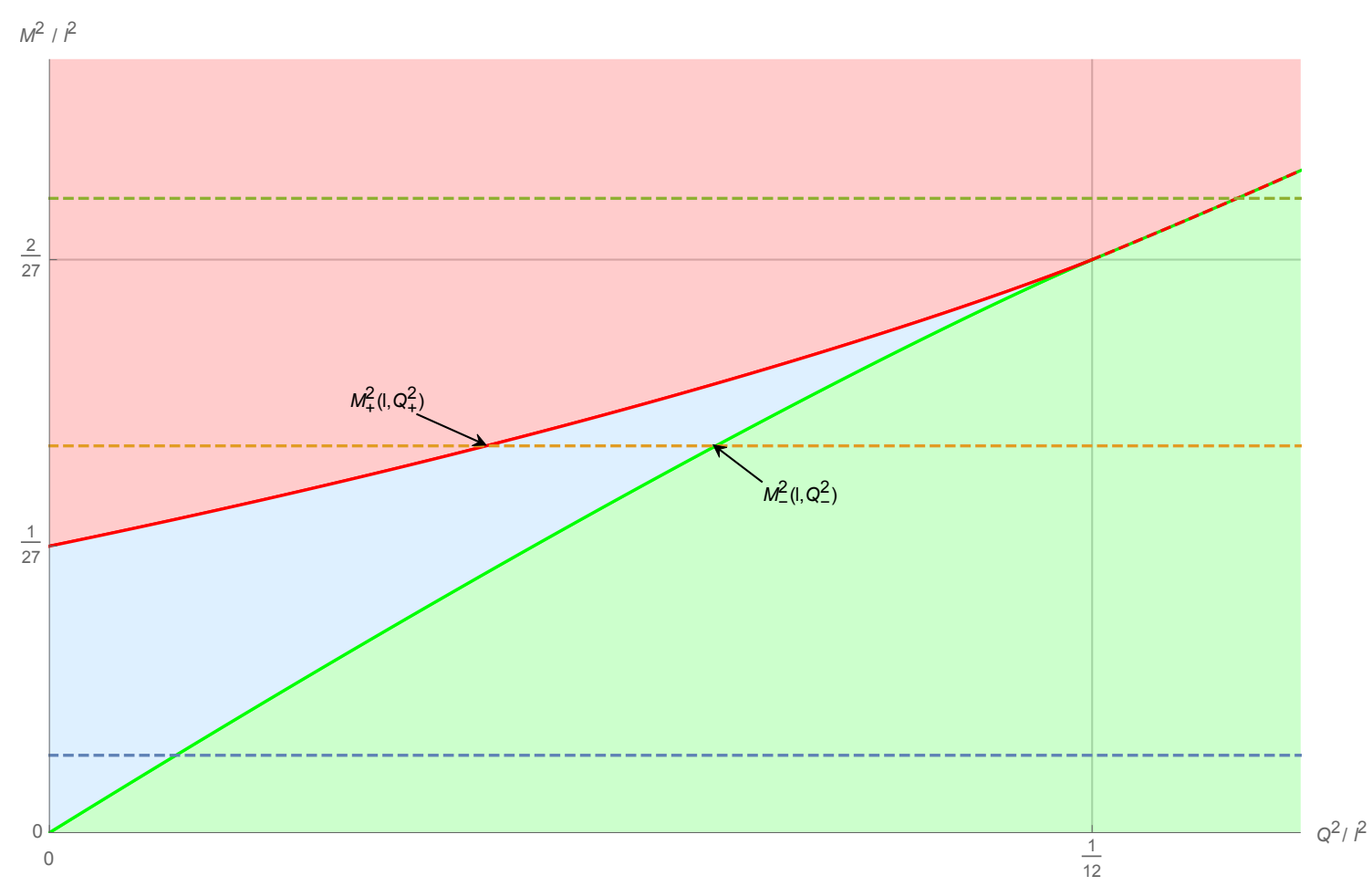

FIGURE 2: The blue filled region corresponds to the range of $M^{2} / l^{2}$ and $Q^{2} / l^{2}$ allowing the black hole to possessing three horizons. The solid red curve corresponds to the maximal mass for given charge, $M^{2}=M_{+}^{2}\left(l, Q_{+}\right)$, where two roots are degenerate $r_{+}=r_{C}$. The solid green curve represents the minimal mass $M^{2}=M_{-}^{2}\left(l, Q_{-}\right)$, where $r_{-}=r_{+}$describing cold extremal black holes. The green filled area corresponds to the region allowed by the de Sitter Weak Gravity Conjecture (dS-WGC) for the (mass, charge) parameters.

- $\left(Q<Q_{-} ; M>M_{-}\left(l, Q^{2}\right)\right): r_{-} \nearrow, r_{+} \searrow$ and $r_{C} \nearrow$ thus the black hole interior measured in Schwarzschild coordinates shrinks, $r_{+} \rightarrow r_{-}$. This continues till a critical value is reached when $Q=Q_{-}$where two horizons degenerate $r_{-}=r_{+}$ (Approaching this region, the $(t, r)$ coordinates are no more appropriate to measure distances, but this is not relevant for our discussion).

- $\left(Q>Q_{-} ; M^{2}<M_{-}^{2}\left(l, Q^{2}\right)\right)$, we have only two regions separated by a (cosmological) horizon. The repulsive electromagnetic interaction is strong enough to forbid the presence of a black hole event horizon.

2. $\frac{l^{2}}{27} \leq M^{2} \leq \frac{2 l^{2}}{27}$ : one has $Q_{+}<Q_{-}$and between $\left[Q_{+}, Q_{-}\right]$one has $M \in\left[M_{-}(l, Q), M_{+}(l, Q)\right]$ thus three horizons (see the red middle horizontal line within the blue area in Fig. 2). Let's vary $Q$ :

- $Q \searrow, r_{-} \searrow, r_{+} \nearrow$ and $r_{C} \searrow \cdot Q \rightarrow Q_{+}$till $Q=Q_{+}$where $r_{+}=r_{C}$. The region III shrinks until it disappears for $Q<Q+$ then, as shown in the red area of Fig. 2 the remaining horizon $r_{-}$separates two regions (I) from the merged $(\mathrm{II}+\mathrm{III}+\mathrm{IV})$ region. The repulsive electrical energy density is too weak to forbid the black hole from eating the de Sitter space-time.

- $Q \nearrow, r_{-} \nearrow, r_{+} \searrow$ and $r_{C} \nearrow$. We start with $Q \rightarrow Q_{-}$, and when we reach $Q=Q_{-}$we have $r_{+}=r_{-}$. For $Q>Q-($ see in Fig. 2 the green area), the region II disappears and the horizon $r_{C}$ separates the merged (I+II+III) regions from region (IV) where $t$ is space-like. The repulsive electromagnetic interaction is strong enough to forbid the presence of a black hole event horizon.

3. $M^{2}=\frac{2 l^{2}}{27}$ : as $Q^{2} \rightarrow l^{2} / 12, r_{-} \rightarrow r_{+} \rightarrow r_{C} \rightarrow l / \sqrt{6}$ correspond to the triple degenerate horizon. Here, the repulsive effects of the electric charge but mainly the cosmological constant are exactly balancing the attractive gravity, and overcome it for $Q>l / \sqrt{12}$ (see Fig. 2).

4. $M^{2}>\frac{2 l^{2}}{27}$ : there is no more black hole, only one apparent horizon remains, on the left or the right of $l / \sqrt{6}$ depending of $(Q, M)$ (see in Fig. 2 the upper horizontal green line). We can write: 


$$
M=\sqrt{\frac{2}{27}} l+\delta M, \quad Q^{2}=\frac{l^{2}}{12}+\sqrt{\frac{2}{3}} \delta Q^{2},
$$

then $\delta M>\delta Q^{2} / l$, describes a giant black hole that swallowed the de Sitter patch (red area in Fig. 2). For $\delta M<\delta Q^{2} / l$, the electromagnetic energy density and a cosmological constant lead to a repulsive force everywhere horizon and there is no black hole (green area in Fig. 2). For $\delta M=\delta Q^{2} / l$, the attractive and repulsive forces are balanced.

We identify here the dS-WGC as the requirement that the repulsive interaction can be strong enough to forbid the appearance of black hole horizons. This leads to:

1. Small charge: $\quad Q^{2} \leq \frac{l^{2}}{12}\left(g^{2} q^{2} \leq \frac{\pi l^{2}}{3 G}\right) \quad$ and $\quad M^{2} \leq \frac{2}{27} l^{2}\left(m^{2} \leq \frac{2}{27 G^{2}} l^{2}\right)$ :

$$
\begin{aligned}
M^{2} & <M_{-}^{2}\left(l, Q^{2}\right)=\frac{1}{54 l}\left[l\left(l^{2}+36 Q^{2}\right)-\left(l^{2}-12 Q^{2}\right)^{3 / 2}\right] \\
& \Longleftrightarrow \quad m^{2}<\frac{1}{54 G^{2} l}\left[\frac{9 G l}{\pi} g^{2} q^{2}+l^{3}-\left(l^{2}-\frac{3 G}{\pi} g^{2} q^{2}\right)^{3 / 2}\right]
\end{aligned}
$$

2. $\underline{\text { Large charge: }} Q^{2} \geq \frac{l^{2}}{12}\left(g^{2} q^{2} \geq \frac{\pi l^{2}}{3 G}\right)$ :

$$
M^{2}<\frac{3}{2} \frac{1}{l^{2}}\left(Q^{2}+\frac{5}{36} l^{2}\right)^{2} \Longleftrightarrow m<\frac{5}{12 \sqrt{6}} \frac{l}{G}+\sqrt{\frac{3}{2}} \frac{g^{2} q^{2}}{4 \pi l} .
$$

\subsection{Small and large curvature limits of the $d S-W G C$ :}

The purpose of the choice of this dS-WGC is that it will provide a continuation of the flat space-time WGC. This can be seen by taking the limit $l \rightarrow \infty$, the sfirst condition in (25) is then empty, while the second reads

$$
M^{2}<Q^{2}-\frac{Q^{4}}{l^{2}}-2 \frac{Q^{6}}{l^{4}}+\mathcal{O}\left(1 / l^{6}\right) \quad \Longleftrightarrow \quad m^{2}<\frac{g^{2} q^{2}}{4 \pi G}-\frac{g^{4} q^{4}}{16 \pi^{2} l^{2}}-\frac{G g^{6} q^{6}}{32 \pi^{3} l^{4}}+O\left(\frac{1}{l^{6}}\right)
$$

where we recognize the well-known WGC in flat space:

$$
g q>\sqrt{4 \pi G} m \quad(l \rightarrow \infty),
$$

and in the corrections due to the presence of a small positive cosmological constant can be written as:

$$
Q^{2}>M^{2}+\frac{M^{4}}{l^{2}}+\mathcal{O}\left(1 / l^{4}\right) \quad \Rightarrow \quad g q>\sqrt{4 \pi G} m\left(1+\frac{G^{2} m^{2}}{2 l^{2}}+\cdots\right) .
$$

In the strong curvature limit, $l \rightarrow 0$, the first condition (24) is empty and the second condition reads

$$
Q^{2}>\sqrt{\frac{2}{3}} l M-\frac{5}{36} l^{2} \Rightarrow Q>\left(\frac{2}{3}\right)^{1 / 4} \sqrt{l M}(1+\mathcal{O}(l)),
$$

which is independent of the Newton constant at leading order:

$$
g q>\left(\frac{32 \pi^{2}}{3}\right)^{1 / 4} \sqrt{\operatorname{lm}} \quad(l \rightarrow 0),
$$

since the factors of $G$ drop, to be compared with (27).

\subsection{Magnetic black hole in de Sitter}

Another possible de Sitter WGC was proposed in [9]. This is based on the condition that given a $U(1)$ gauge theory, the size of the minimally charged monopole should lie in between the event and cosmological horizons. This size is of order $1 / \Lambda_{m}$, with $\Lambda_{m}$ the cut-off of the theory, while the monopole mass is $\Lambda_{m} / g$ with $g$ the gauge coupling. The magnetic Reissner-Nordstrøm black hole associated with this monopole has a metric (11):

$$
f(r)=1-\frac{2 \Lambda_{m}}{g^{2} r}+\frac{q_{m}^{2}}{g^{2} r^{2}}-\frac{r^{2}}{l^{2}},
$$


where $q_{m}$ is the magnetic charge (see (8)). The monopole charge was taken to vanish in [9], $q_{m}=0$. The condition on the size of the monopole lies is required to satisfy:

$$
f\left(\frac{1}{\Lambda_{m}}\right)=1+\frac{\Lambda_{m}^{2}}{g^{2}}\left(q_{m}^{2}-2\right)-\frac{1}{\Lambda_{m}^{2} l^{2}} \geq 0 .
$$

This can be written as:

$$
\frac{\left(2-q_{m}^{2}\right) l^{2}}{g^{2}} \Lambda_{m}^{4}-l^{2} \Lambda_{m}^{2}+1 \leq 0 .
$$

which means that the discriminant of the quadratic polynomial in $\Lambda_{m}^{2}$ is positive:

$$
g \geq \frac{2 \sqrt{\left(2-q_{m}^{2}\right)}}{l} \quad \underset{q_{m}=1}{\longrightarrow} \quad g \geq \frac{2}{l},
$$

for the minimal charge. Note that the extremal case, $g=\frac{2}{l}$ corresponds to a magnetic monopole with a mass $M_{m}=l / \sqrt{8}$ and size o $1 / \Lambda_{m}=l / \sqrt{2}=r_{C}$.

Comparing with [9], we see that their condition looks like (34) but with a $\sqrt{2}$. More important, it makes an implicit assumption about the mass dependence through the de Sitter radius. In fact, the values of $\left(M_{m}^{2}, Q_{m}^{2}\right)=\left(l^{2} / 8, l^{2} / 4\right)$ are in the region described by the condition (25) and our stronger conditions (24) and (25) insure that the condition (34) is satisfied.

\section{THE $U(1)$ R-SYMMETRY CASE:}

In $N=1$ supergravity, a de Sitter background can be obtained after gauging a $U(1)_{R}[14]$ gauge R-symmetry through a positive contribution from Fayet-Iliopoulos D-term. A minimal toy model is given in [15] where pure supergravity is coupled to one vector multiplet gauging the R-symmetry. It was shown in [16] that the dual theory is a supergravity theory with deformed supersymmetry, i.e. with a magnetic Fayet-Iliopoulos term [16].

Since both a cosmological constant $\Lambda$ and a charge $q_{R}$ of the fermions (gravitino and gaugino) result from the unique gauging of the R-symmetry, there are not independent [16] (using $\kappa=1$ ):

$$
\Lambda=2 q_{3 / 2}^{2} \Rightarrow q_{3 / 2}^{2}=\frac{3}{2 l^{2}} \quad \text { and } \quad Q_{3 / 2}^{2}=\frac{3}{64 \pi^{2} l^{2}}
$$

here $q_{3 / 2}$ stands fo the physical R-charge of the gravitino: $q_{3 / 2}=q g$. This is dual to a minimal magnetic charge $\tilde{q}_{3 / 2}=2 \pi / q_{3 / 2}$, i.e. $\tilde{Q}_{3 / 2}^{2} / l^{2}=1 /\left(8 q_{3 / 2}^{2} l^{2}\right)=1 / 12$, the limiting value in Fig. 2 . The gravitino (and gaugino) mass term in the Lagrangian vanishes as in this simplest model the superpotential is zero. These lead to:

$$
\frac{Q_{3 / 2}^{2}}{l^{2}}=\frac{3}{64 \pi^{2} l^{4}} \Rightarrow\left\{\frac{Q_{3 / 2}^{2}}{l^{2}} \underset{l \rightarrow+\infty}{\longrightarrow} 0 ; \quad \frac{Q_{3 / 2}^{2}}{l^{2}} \underset{l \rightarrow 0}{\longrightarrow} \infty\right\}
$$

therefore taking all positive values on the horizontal axis of Fig. 2.

Let's suppose that the gravitino, if it plays the role of the dS-WGC state. Its maximal squared-mass is then (25) and (24) for small and large $l$, respectively. The two formulae overlap for $l=\sqrt{3 / 4 \pi}$, i.e. $\Lambda=4 \pi, Q_{3 / 2}^{2}=l^{2} / 12=1 / 16 \pi$ and $M_{3 / 2}^{2}=1 / 18 \pi$. This reads:

- For $l \in] 0, \sqrt{3 / 4 \pi}], 1 M_{3 / 2}^{2} \in\left[1 / 18 \pi,+\infty\right.$ [ and the maximal squared-mass goes as $1 / l^{6}$ for small $l$.

- For $l \in\left[\sqrt{3} / \sqrt{4 \pi},+\infty\left[\right.\right.$, the maximal squared-mass $\left.\left.M_{3 / 2}^{2}=M_{3 / 2-}^{2}\left(l, Q_{3 / 2}^{2}\right) \in\right] 0,1 / 18 \pi\right]$ behaves as $1 / l^{2}$ for large $l$, a behaviour reminiscent of the $m_{3 / 2}^{2} M_{P l}^{2}$ to the vacuum energy in $N=1$ supergravity.

If the gravitino is instead the dS-WGC-state of the magnetic theory, then:

$$
\frac{\Lambda_{m}}{g^{2}} \leq \sqrt{\frac{2}{27}} l \quad \text { and } \quad \frac{1}{g^{2}}=\frac{2}{3} l^{2} \Rightarrow \Lambda_{m} \leq l / \sqrt{6}=r_{C},
$$

which can also be written:

$$
\Lambda_{m} \leq\left(r_{C} M_{P l}\right) M_{P l} \quad \Leftrightarrow \quad \Lambda_{m} \leq \frac{M_{P l}}{\sqrt{2 \Lambda}} M_{P l} .
$$

Here $r_{C}$ stands now for the cosmological horizon in the magnetic dual theory,

We introduce now charged chiral multiplets with scalars $\phi_{i}$ having physical R-charges $q_{i}$. This allows for a D-term potential:

$$
V_{D}=\frac{1}{2}\left(\sum_{i} q_{i}\left|\phi_{i}\right|^{2}+2 q_{3 / 2}\right)^{2} \text {, }
$$


The scalar masses $m_{i}$ of $\phi_{i}$ read:

$$
m_{i}^{2}=2 q_{i} q_{3 / 2}
$$

which implies:

$$
Q_{i}^{2}=\frac{q_{i}^{2}}{32 \pi^{2}} \quad ; \quad M_{i}^{2}=\frac{m_{i}^{2}}{64 \pi^{2}}=\frac{q_{i} q_{3 / 2}}{32 \pi^{2}} .
$$

One can add a simple quadratic superpotential $W: W=\frac{\mu}{2} \phi_{0}^{2}$ or $W=\mu \phi_{+} \phi_{-}$for some fields $\phi_{0,+,-}$, where $\mu$ is a mass parameter. Then $q_{0}=q_{3 / 2}$ or $q_{+}+q_{-}=2 q_{3 / 2}$, giving:

$$
Q_{0}^{2}=Q_{3 / 2}^{2}=M_{0}^{2}
$$

and

$$
Q_{+}+Q_{-}=2 Q_{3 / 2} \quad ; \quad M_{ \pm}^{2}=Q_{ \pm} Q_{3 / 2}+\mu^{2}
$$

It is obvious that (42) can not provide a dS-WGC state while (43) has two extrema:

$$
\left\{Q_{ \pm}=2 Q_{3 / 2}, \quad Q_{\mp}=0\right\} \quad ; \quad\left\{M_{ \pm}^{2}=2 Q_{3 / 2}^{2}+\mu^{2}, \quad M_{\mp}^{2}=\mu^{2}\right\},
$$

which to be a dS-WGC state require

$$
\begin{aligned}
& Q_{3 / 2}^{2} \leq \frac{1}{32 \pi} \quad: \quad l \geq \sqrt{\frac{3}{2 \pi}} \quad \Rightarrow \quad \mu^{2}<\frac{16 \pi^{3} l^{4}-2 \sqrt{\frac{\left(4 \pi^{2} l^{4}-9\right)^{3}}{l^{8}} l^{2}+27 \pi}}{864 \pi^{3} l^{2}} \\
& Q_{3 / 2}^{2} \geq \frac{1}{16 \pi} \quad: \quad l \leq \sqrt{\frac{3}{4 \pi}} \quad \Rightarrow \quad \mu^{2}<\frac{27}{512 \pi^{4} l^{6}}-\frac{1}{64 \pi^{2} l^{2}}+\frac{25 l^{2}}{864}
\end{aligned}
$$

from (24) and (25), respectively. The maximally charged fermion has the sea mass and charge as the gravitino discussed above and is therefore subject to the same constraints.

\section{CONCLUSIONS}

If the the swampland program and the Weak Gravity Conjecture have to be applied in real world, then they should begged approximation in the presence of a small non-vanishing vacuum energy as our Universe falls in this class. How does the Weak Gravity Conjecture (WGC) get modified by a positive vacuum energy, for instance a cosmological constant? This the question raised and answered in [1].

We have discussed the simplest case of de Sitter spacetime and obtained explicit form for the dS-WGC. We have checked that the well known flat space-time limit is reproduced when the vacuum energy is taken to be negligible. We have only scratched the surface of explicit checks of this conjecture in quantum gravity theories as explicit examples of de Sitter vacua in supergravity are difficult to construct, not to say totally absent in string theory. In the future, it will be interesting to understand the application of our conjecture for cosmological models.

\section{ACKNOWLEDGEMENTS}

The research of I.A. was partially performed as International professor of the Francqui Foundation, Belgium. The work of K.B. is supported by the Agence Nationale de Recherche under grant ANR-15-CE31-0002 "HiggsAutomator",.

\section{References}

[1] I. Antoniadis and K. Benakli, Fortsch. Phys. 68 (2020) no.9, 2000054 doi:10.1002/prop.202000054 [arXiv:2006.12512 [hep-th]].

[2] C. Vafa, "The String landscape and the swampland," hep-th/0509212; H. Ooguri and C. Vafa, "On the Geometry of the String Landscape and the Swampland," Nucl. Phys. B 766 (2007) 21 [hep-th/0605264]. For a review see e.g.: E. Palti, "The Swampland: Introduction and Review," Fortsch. Phys. 67 (2019) no.6, 1900037 [arXiv:1903.06239 [hep-th]] and references therein.

[3] N. Arkani-Hamed, L. Motl, A. Nicolis and C. Vafa, "The String landscape, black holes and gravity as the weakest force," JHEP 0706 (2007) 060 [hep-th/0601001].

[4] J. M. Maldacena and C. Nunez, "Supergravity description of field theories on curved manifolds and a no go theorem," Int. J. Mod. Phys. A 16 (2001) 822 [hep-th/0007018].

[5] U. H. Danielsson and T. Van Riet, Int. J. Mod. Phys. D 27 (2018) no.12, 1830007 [arXiv:1804.01120 [hep-th]].

[6] G. Obied, H. Ooguri, L. Spodyneiko and C. Vafa, "De Sitter Space and the Swampland," arXiv:1806.08362 [hep-th]; S. K. Garg and C. Krishnan, JHEP 11 (2019), 075 [arXiv:1807.05193 [hep-th]]; D. Andriot, "On the de Sitter swampland criterion," Phys. Lett. B 785, 570 (2018) [arXiv:1806.10999 [hep-th]]; H. Ooguri, E. Palti, G. Shiu and C. Vafa, "Distance and de Sitter conjectures on the Swampland," Phys. Lett. B 788, 180 (2019) [arXiv:1810.05506 [hep-th]]. 
[7] I. Antoniadis, Y. Chen and G. K. Leontaris, “Perturbative moduli stabilisation in type IIB/F-theory framework," Eur. Phys. J. C 78 (2018) no.9, 766 [arXiv:1803.08941 [hep-th]]; "Logarithmic loop corrections, moduli stabilisation and de Sitter vacua in string theory," JHEP 2001 (2020) 149 [arXiv:1909.10525 [hep-th]].

[8] M. Montero, T. Van Riet and G. Venken, “Festina Lente: EFT Constraints from Charged Black Hole Evaporation in de Sitter," JHEP 01 (2020), 039 [arXiv:1910.01648 [hep-th]].

[9] Q. G. Huang, M. Li and W. Song, "Weak gravity conjecture in the asymptotical dS and AdS background," JHEP 10 (2006), 059 [arXiv:hepth/0603127 [hep-th]].

[10] F. Kottler, "Uber die physikalischen Grundlagen der Einsteinschen Gravitationstheorie", Ann. Phys. (Leipzig) 56 (1918) 410; D. Kramer, H. Stepani, E. Herlt, M. MacCallum, Exact solutions of Einstein???s field equations (Cambridge University Press, Cambridge, 1980 ). 126.

[11] L. Romans, "Supersymmetric, cold and lukewarm black holes in cosmological Einstein-Maxwell theory," Nucl. Phys. B 383 (1992), $395-415$ [arXiv:hep-th/9203018 [hep-th]].

[12] H. Nariai, "On some static solutions of Einstein's gravitational field equations in a spherically symmetric case," Gen. Rel. Grav. 31 (1999) 951.

[13] P. H. Ginsparg and M. J. Perry, "Semiclassical Perdurance of de Sitter Space," Nucl. Phys. B 222 (1983), 245-268.

[14] D. Z. Freedman and A. Van Proeyen, "Supergravity," Cambridge University Press, Cambridge, 2012.

[15] D. Z. Freedman, "Supergravity with axial gauge invariance," Phys. Rev. D 15 (1977) 1173

[16] I. Antoniadis, J. P. Derendinger, H. Jiang and G. Tartaglino-Mazzucchelli, "Magnetic Deformation of Super-Maxwell Theory in Supergravity," arXiv:2005.11374 [hep-th].

[17] T. Crisford and J. E. Santos, Phys. Rev. Lett. 118 (2017) no.18, 181101 [arXiv:1702.05490 [hep-th]]; T. Crisford, G. T. Horowitz and J. E. Santos, "Testing the Weak Gravity - Cosmic Censorship Connection," Phys. Rev. D 97 (2018) no.6, 066005 [arXiv:1709.07880 [hep-th]]; G. T. Horowitz and J. E. Santos, "Further evidence for the weak gravity ? cosmic censorship connection," JHEP 06 (2019), 122 [arXiv:1901.11096 [hep-th]]. 